\title{
Targeted Single-Site MOF Node Modification: Trivalent Metal Loading via Atomic Layer Deposition
}

\author{
In Soo Kim, ${ }^{\dagger}$ Joshua Borycz, $^{\ddagger}$ Ana E. Platero-Prats, ${ }^{\S}$ Samat Tussupbayev, ${ }^{\ddagger}$ Timothy C. Wang,
} Omar K. Farha, ${ }_{\|, \perp}{ }^{\prime}$ Joseph T. Hupp, ${ }^{\dagger, \|}$ Laura Gagliardi, ${ }^{\ddagger}$ Karena W. Chapman, ${ }^{\S}$ Christopher J. Cramer, $*, \neq$ and Alex B. F. Martinson*, ${ }^{\dagger}$

\author{
${ }^{\dagger}$ Materials Science Division, and ${ }^{\S}$ X-ray Science Division, Advanced Photon SourceArgonne National Laboratory, 9700 S. Cass \\ Avenue, Argonne, Illinois 60439, United States \\ ${ }^{\ddagger}$ Department of Chemistry, Supercomputing Institute, and Chemical Theory Center, University of Minnesota, Minneapolis, \\ Minnesota 55455, United States \\ "Department of Chemistry, Northwestern University, 2145 Sheridan Road, Evanston, Illinois 60208, United States \\ ${ }^{\perp}$ Department of Chemistry, Faculty of Science, King Abdulaziz University, Jeddah, Saudi Arabia
}

\section{Supporting Information}

ABSTRACT: Postsynthetic functionalization of metal organic frameworks (MOFs) enables the controlled, high-density incorporation of new atoms on a crystallographically precise framework. Leveraging the broad palette of known atomic layer deposition (ALD) chemistries, $\underline{\mathrm{ALD}}$ in $\underline{\mathrm{MOFs}}$ (AIM) is one such targeted approach to construct diverse, highly functional, fewatom clusters. We here demonstrate the saturating reaction of trimethylindium $\left(\mathrm{InMe}_{3}\right)$ with the node hydroxyls and ligated water of NU-1000, which takes place without significant loss of MOF crystallinity or internal surface area. We computationally identify the elementary steps by which trimethylated trivalent metal compounds (ALD precursors) react with this Zr-based MOF node to generate a uniform and well characterized new surface layer on the node itself,

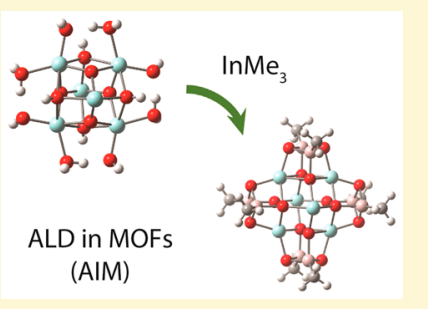
and we predict a final structure that is fully consistent with experimental X-ray pair distribution function (PDF) analysis. We further demonstrate tunable metal loading through controlled number density of the reactive handles $\left(-\mathrm{OH}\right.$ and $\left.-\mathrm{OH}_{2}\right)$ achieved through node dehydration at elevated temperatures.

\section{INTRODUCTION}

Metal-organic frameworks (MOFs) are attractive systems for catalytic studies, ${ }^{1,2}$ in part because they exhibit uniform mesoscale and/or microscale porosity, generated by periodic and atomically well-defined structures. Importantly, these structures, comprising organic linkers and inorganic, metalcontaining nodes, are amenable to full experimental structural characterization and rigorous computational modeling. ${ }^{3-6}$ Although the vast majority of MOF catalysis work focuses on linkers as catalysts ${ }^{7}$ for condensed-phase reactions, a few reports of node-based catalysis have also appeared, ${ }^{8-16}$ including at least one for high temperature $\left(350{ }^{\circ} \mathrm{C}\right)$ heterogeneous catalysis of a gas-phase oxidation reaction. ${ }^{17}$ Increasingly popular as nodes for these types of studies are hexa-zirconium(IV)oxo/hydroxo/aqua species and their hafnium(IV) analogues. The lability and reactivity of the nodes' oxygen-rich ligands-specifically, aqua and terminal hydroxo ligands-allows for site-specific functionalization, both with nonstructural organic ligands ${ }^{18-22}$ and, in principle, with nonstructural metal ions. We have shown that the latter can be accomplished in reproducible fashion with a suitably porous MOF, NU-1000 (aperture width $>3 \mathrm{~nm}$ ), via chemistry akin to atomic-layer deposition (ALD), a materials synthesis technique typically applied to hydroxyl-presenting surfaces of purely inorganic materials. ${ }^{23-25}$ We reasoned that the nodes within
NU-1000 and related MOFs could be viewed chemically as tiny fragments of zirconia, and therefore, be subject to the selflimiting, conformal functionalization chemistry that characterizes conventional ALD. ${ }^{26}$ Indeed, with vapor-phase organometallic precursors such as diethyl zinc, and as previously described in preliminary fashion, ALD in MOFs (AIM) ${ }^{26}$ is readily accomplished specifically with NU-1000. Here, with the ultimate goal of arbitrary, single-atom precision for MOF postmetalation, we investigate and report on the details of AIM using two well-known ALD precursors, trimethylindium $\left(\mathrm{InMe}_{3}\right)$ and trimethyaluminum $\left(\mathrm{AlMe}_{3}\right)$.

Recent advances in both MOF and ALD science make their potential union both feasible and versatile. While the stability ranges of early MOFs and process ranges of early ALD procedures occupied different temperature windows-generally below and above $200{ }^{\circ} \mathrm{C}$, respectively-advances in both fields now permit significant overlap of these ranges. For example, selected MOFs based on $\mathrm{Zr}_{6} \mathrm{O}_{16}$ nodes (Figure 1), retain their mesoporosity at temperatures above $400{ }^{\circ} \mathrm{C}$, and in some case above $500{ }^{\circ} \mathrm{C} .{ }^{12,26,27}$ In addition, an ever growing number of ALD processes for oxides, sulfides, nitrides, and pure metals

Received: April 27, 2015

Revised: June 19, 2015

Published: July 2, 2015 
a)

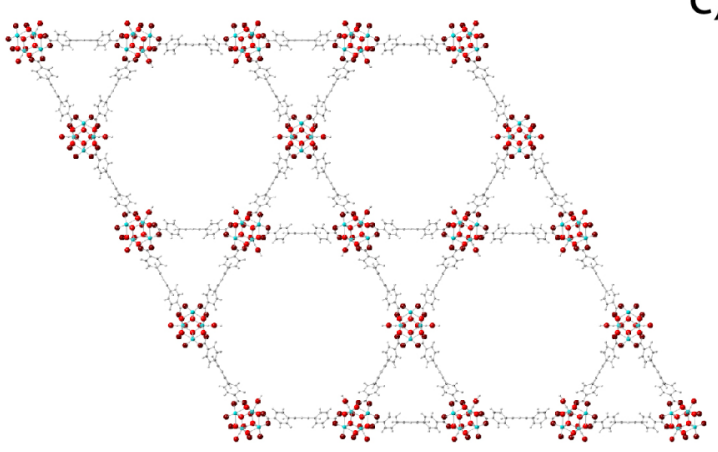

b)

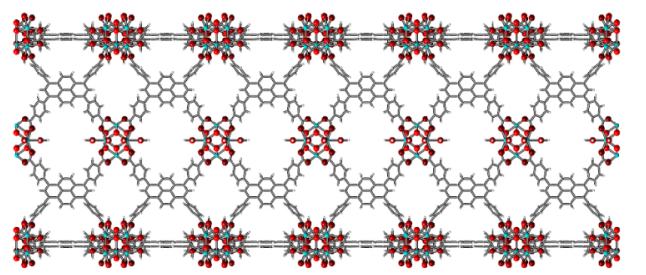

C)

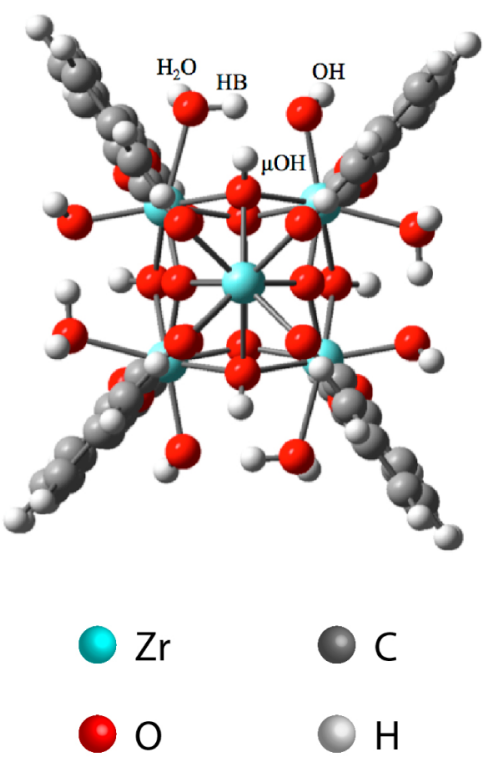

Figure 1. (a, b) $\mathrm{Zr}_{6}$-based framework NU-1000. (c) Cluster used to model the $\mathrm{Zr}_{6}$-node in NU-1000.

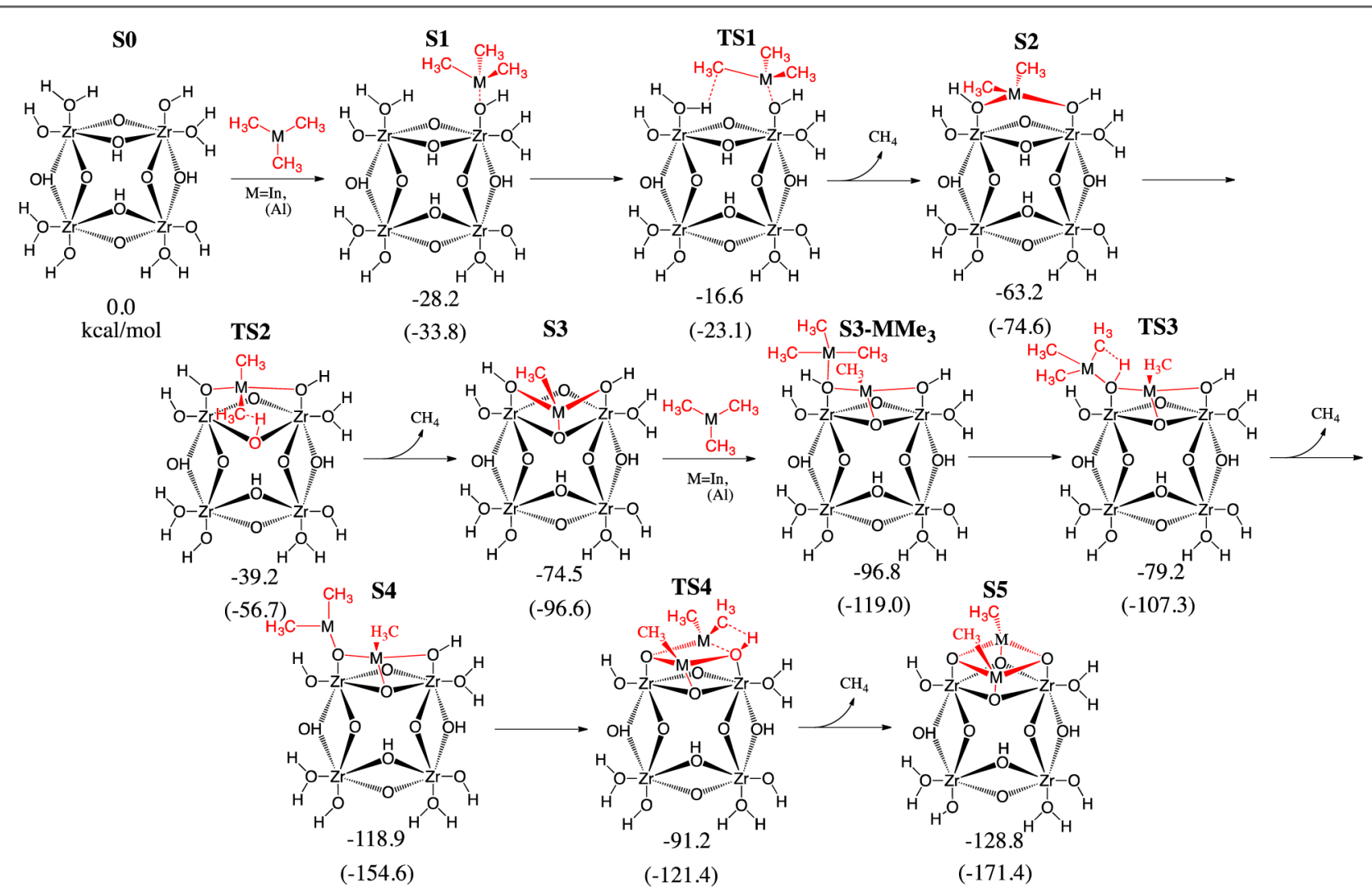

Figure 2. M06-L relative enthalpies $\left(\mathrm{kcal} \mathrm{mol}^{-1}\right)$ for intermediates and TS structures on the reaction coordinate for one face of the NU-1000 node reacting with 2 equivalents of $\mathrm{AlMe}_{3}$ or $\mathrm{InMe}_{3}$ (in parentheses) and liberating 4 equivalents of $\mathrm{CH}_{4}$. Subsequent reaction coordinates for the remaining faces of the node (not shown) were found to be entirely equivalent, with negligible differences in enthalpies relative to analogous precursors, i.e., there is no significant influence of one face upon another over the course of reaction.

have been identified at temperatures as low as $100{ }^{\circ} \mathrm{C} .^{28-32}$ Appropriately chosen MOFs exhibit the high porosity and large pore apertures $(>3 \mathrm{~nm})$ desirable to minimize the influence of mass transport limitations that would otherwise practically inhibit vapor-phase loading. Such characteristics allow for AIM, where precise surface chemical control is retained through self- limiting reactions that allow uniform reaction throughout an arbitrarily complex framework. Recently, the platform MOF material NU-1000 has been modified with $\mathrm{Al}, \mathrm{Zn}$, and their combination. ${ }^{26}$ To provide mechanistic insight and establish universal applicability of the AIM approach, we take trimethylated trivalent metal precursors as model reactants, 
and examine the extent to which uniform and self-limiting (ALD-like) reactions can be achieved in MOFs. Furthermore, we determine the exact locations of the trivalent metals, obtaining results that are consistent with a mechanism that we derive from first-principles computation. Finally, we assess one of the most chemically aggressive ALD precursors, $\mathrm{AlMe}_{3}$, for compatibility with the MOF framework.

\section{RESULTS AND DISCUSSION}

A $\mathrm{Zr}_{6}$-based framework, NU-1000's nodes are octahedral $\mathrm{Zr}_{6}$ clusters including four each bridging $\mu-\mathrm{O}$ and $\mu-\mathrm{OH}$ groups. The 3D structure of the MOF results from bridging of the nodes by tetra-carboxylate linkers (Figure 1a, b) ${ }^{26}$ Importantly, under ambient conditions, four each aquo and hydroxo groups can occupy remaining open metal coordination sites of the node. The detailed structure of NU-1000 was previously investigated both computationally and experimentally to reveal a staggered mixed proton topology for the $\left[\mathrm{Zr}_{6}\left(\mu_{3}-\mathrm{O}\right)_{4}\left(\mu_{3}-\right.\right.$ $\left.\mathrm{OH})_{4}(\mathrm{OH})_{4}\left(\mathrm{H}_{2} \mathrm{O}\right)_{4}\right]^{8+}$ cluster (Figure 1c) at room temperature. $^{33}$

The $-\mathrm{OH}$ and $-\mathrm{OH}_{2}$ functional groups at the surface of the node present in a manner making them likely to be amenable to many conventional oxide ALD half-reactions. Using density functional theory (DFT) calculations, we have identified highly exothermic reaction pathways for reaction of the node with two trimethylated trivalent metal compounds, $\mathrm{AlMe}_{3}$ and $\mathrm{InMe}_{3}$, as illustrated in Figure 2. This chemistry is in close analogy to that previously hypothesized for planar surface modification in traditional thin film ALD growth. ${ }^{34}$ On the basis of the reaction pathways, a maximum of 8 metal atoms are predicted to bind to each $\mathrm{Zr}_{6}$ node with the release of up to 12 methane $\left(\mathrm{CH}_{4}\right)$ molecules.

Computational Results. There are four chemically distinct protons on each of the four faces of the $\mathrm{Zr}_{6}$-node in NU-1000, one that engages in a hydrogen bond between the aquo and hydroxo ligands ( $\mathrm{HB}$; see Figure 1c), two that are nonhydrogen bonded on the aquo $\left(\mathrm{H}_{2} \mathrm{O}\right)$ and hydroxo $(\mathrm{OH})$ ligands, respectively, and one that is on the core itself $(\mu \mathrm{OH})$. To test which of the four protons on each face is most likely to be replaced with either $\mathrm{AlMe}_{3}$ or $\mathrm{InMe}_{3}$, each proton was individually substituted for an $\mathrm{AlMe}_{2}$ or $\mathrm{InMe}_{2}$ group and the structure of the cluster was then optimized with fixed linker positions. Replacement of hydrogen $\mathrm{HB}$ with $\mathrm{AlMe}_{2}$ or $\mathrm{InMe}_{2}$ results in the most stable product (Table 1 ). We subsequently located the transition-state (TS) structure leading to this intermediate, and we characterized all of the elementary steps involved in the further reaction of the node face under operating conditions. The full reaction coordinate, shown in Figure 2, indicates that each face of the $\mathrm{Zr}_{6}$-node will react with

Table 1. Relative Reaction Energies for Substitution of $\mathrm{AlMe}_{2}$ and $\mathrm{InMe}_{2}$ (and Generation of $\mathrm{CH}_{4}$ ) at the Positions of the Labeled Hydrogens in the Bare $\mathrm{Zr}_{6}$ Cluster Shown in Figure 1c

\begin{tabular}{lccccc} 
& \multicolumn{4}{c}{ relative energy $\left(\mathrm{kcal} \mathrm{mol}^{-1}\right)$} \\
\cline { 2 - 3 } \cline { 5 - 6 } \cline { 5 - 6 } & \multicolumn{3}{c}{$\Delta E$} & & \multicolumn{3}{c}{$\Delta G$} \\
\cline { 2 - 5 } & $\mathrm{AlMe}_{3}$ & $\mathrm{InMe}_{3}$ & & $\mathrm{AlMe}_{3}$ & $\mathrm{InMe}_{3}$ \\
$\mathrm{HB}$ & 0.0 & 0.0 & & 0.0 & 0.0 \\
$\mathrm{H}_{2} \mathrm{O}$ & 18.5 & 15.7 & & 19.2 & 17.3 \\
$\mathrm{OH}$ & 22.3 & 21.6 & & 22.9 & 23.0 \\
$\mu \mathrm{OH}$ & 24.4 & 22.6 & & 24.9 & 24.0
\end{tabular}

two ALD precursors to generate four $\mathrm{CH}_{4}$ product molecules, i.e., our model predicts that every $\mathrm{O}-\mathrm{H}$ bond on each node will react with $\mathrm{AlMe}_{3}$ and $\mathrm{InMe}_{3}$ to lead to a final structure with eight tetrahedrally coordinated $(\mathrm{RO})_{3} \mathrm{AlMe}$ or $(\mathrm{RO})_{3} \mathrm{InMe}$ groups disposed at the surface.

Experimental Results. To verify the self-limiting nature of the precursor with hydroxyl and water groups, we undertook a full saturation study including exploration of mole-limited and diffusion-time limited variable space (Figure 3). In each experiment, $10 \mathrm{mg}$ of microcrystalline NU-1000 powder was warmed to $125{ }^{\circ} \mathrm{C}$ under vacuum with 0.5 Torr of flowing nitrogen for $20 \mathrm{~min}$ in order to remove any physisorbed water or solvent. Next, the powder was soaked at the desired temperature (75 and $125{ }^{\circ} \mathrm{C}$ for $\mathrm{InMe}_{3}$ and $\mathrm{AlMe}_{3}$, respectively) prior to a $60 \mathrm{~s}$ exposure to water vapor. Further soaking under flowing $\mathrm{N}_{2}$ at temperature establishes an equilibrium $-\mathrm{OH}$ and $-\mathrm{OH}_{2}$ population. Finally, the metal precursor $\left(\mathrm{InMe}_{3}\right.$ or $\left.\mathrm{AlMe}_{3}\right)$ was delivered to the reaction chamber under its own vapor pressure and exposed without pumping (quasi-static mode) for the indicated time for a given number of repetitions. The results of the AIM process with $\mathrm{InMe}_{3}$ (Figure 3) reveal many of the characteristics of archetypal ALD surface reactions, albeit with much larger doses and exposures, as predicted for a large surface area powder with high aspect ratio pores. Clearly, a stoichiometric number of precursor molecules must be delivered with respect to the available reaction sites in order to achieve a saturating reaction. The number of sites in $10 \mathrm{mg}$ of powder is estimated to be $3.7 \times 10^{-5}$ moles based on 8 sites per $\mathrm{Zr}_{6}$ node. On the basis of the vapor pressure of $\mathrm{InMe}_{3}$, an equal or greater number of moles are expected to be delivered in a single $0.4 \mathrm{~s}$ dose. Therefore, under these processing conditions, the reaction is never mole-limited. Still, because of the very high aspect ratio of the channels through which the vapor is required to traverse $(\sim 10000)$, a nontrivial time for precursor diffusion to all points within the microcrystals must also be considered. An empirical formula ${ }^{35}$ based on Monte Carlo simulations assuming Knudsen diffusion was used to calculate a total exposure time of at least $20 \mathrm{~s}$ required to traverse the long, narrow channels with this aspect ratio. This is roughly consistent with a $600 \mathrm{~s}$ exposure time required to achieve the complete loading of the longest crystals. As evidenced by the saturating In ratio in the limit of large dose and long diffusion time, the process shows ideal self-limiting behavior. Based on the saturation studies under these optimized ALD conditions, the number of In per $\mathrm{Zr}_{6}$ node obtained by inductively coupled plasma-optical emission spectroscopy (ICP-OES) saturates at $\sim 6\left(\mathrm{In}: \mathrm{Zr}_{6}=1\right)$, in reasonable agreement with the computational prediction. Several factors account for the deviation from the computationally predicted loading of 8 In per $\mathrm{Zr}_{6}$ node. First, the NU-1000 structure is known to contain a fraction (perhaps 25\%) of "secondary" nodes within the largest pores. ${ }^{26}$ This secondary node is connected to the framework through additional linkers, each one of which reduces the potential number of deposition sites on neighboring nodes by 2 . Furthermore, the computationally predicted reaction assumes full initial ligation of the node $\left(4 \mathrm{OH}_{2}, 4 \mathrm{OH}, 4 \mu-\mathrm{OH}\right)$, which may be slightly reduced via dehydration under continuous heating $\left(75^{\circ} \mathrm{C}\right)$ in vacuum. Finally, there is the possibility that only a subset of the potential reaction sites are reasonably accessible to $\mathrm{InMe}_{3}$. This possibility, however, was minimized by performing saturation studies in excess of both diffusion time and moles of $\mathrm{InMe}_{3}$ (Figure 3). Furthermore, the 

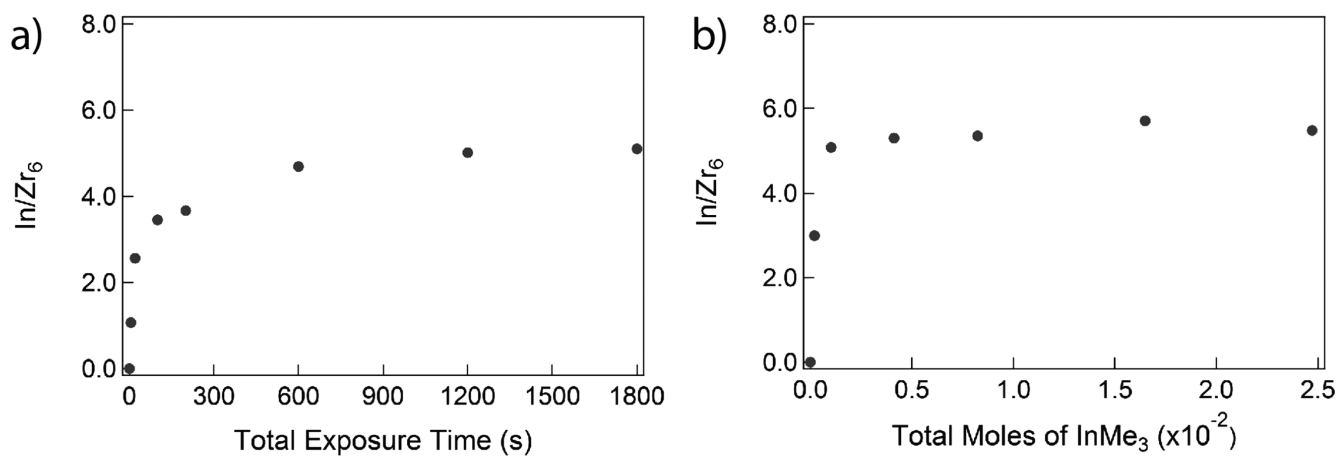

Figure 3. In to $\mathrm{Zr}_{6}$ node ratio as a function of the ALD process conditions. Subsaturating In loading is observed upon delivery of a nonstoichiometric $\mathrm{InMe}_{3}$ dose or insufficient exposure time. In the limit of exceptionally large and long exposures, the process exhibits self-limiting behavior.
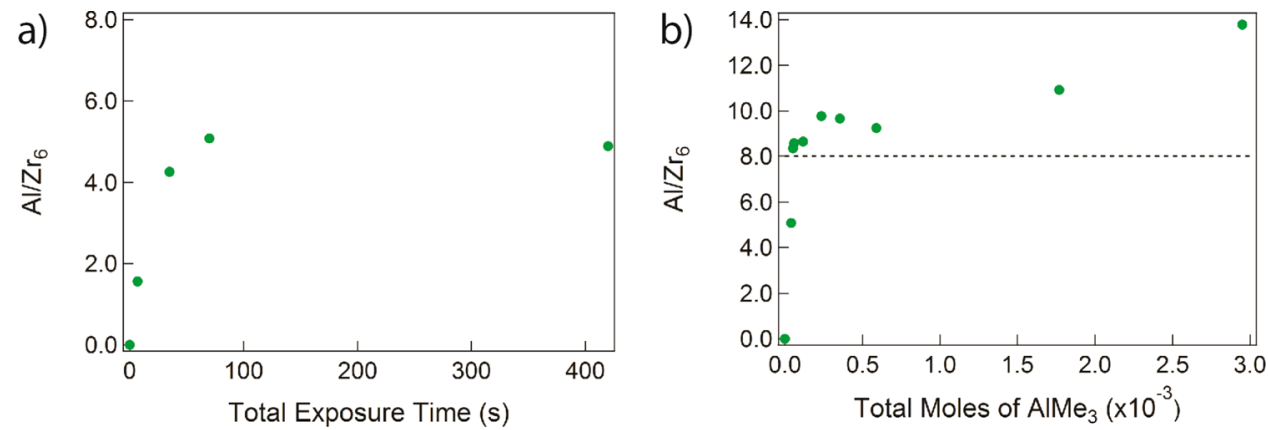

Figure 4. ALD metal to $\mathrm{Zr}_{6}$ node ratio as a function of ALD process conditions. The delivery of a nonstoichiometic AlMe $\mathrm{A}_{3}$ dose or insufficient diffusion time produce subsaturating $\mathrm{Al}$ loading. In the limit of exceptionally large and long doses, the process does not exhibit self-limiting behavior.

possibility of a minority of clogged pores, through which the metal precursor will never pass, has not been rigorously excluded.

Although the AIM process with $\mathrm{AlMe}_{3}$ shows, with moderate exposures, apparent self-limiting behavior with $\sim 6 \mathrm{Al}$ per $\mathrm{Zr}_{6}$ (Figure $4 \mathrm{a}$ ), larger exposures reveal non-self-limiting behavior $\left(\sim 14\right.$ metal per $\mathrm{Zr}_{6}$ node) as shown in Figure $4 \mathrm{~b}$.

Consistent with its strong Lewis acidity, $\mathrm{AlMe}_{3}$ is known to aggressively react with many functional groups beyond $-\mathrm{OH}$. In extreme cases, for example, $\mathrm{AlMe}_{3}$ preferentially attacks and abstracts oxygen anions from surface oxides of GaAs and $\mathrm{In}_{0.2} \mathrm{Ga}_{0.8}$ As substrates. ${ }^{36,37}$ As such, reaction with components in the MOF framework beyond the hydroxylated node is not extraordinary. The extra-nodal reactivity of this aggressive precursor is further corroborated by the significant loss of sample crystallinity evident in powder X-ray diffraction (PXRD) data obtained following large $\mathrm{AlMe}_{3}$ exposures, Figure $\mathrm{S} 1$ in the Supporting Information. Although $\mathrm{InMe}_{3}$ is also Lewis acidic (albeit not as much as $\mathrm{AlMe}_{3}$ ), the strong diffraction peaks of the parent MOF are retained even after total exposures in excess of $\sim 2 \mathrm{~h}$. Furthermore, the large specific surface area that is associated with the mesoporosity of $\mathrm{NU}-1000$ is significantly degraded upon large $\mathrm{AlMe}_{3}$ exposures, whereas the surface area is retained even after exposure to a 10fold larger $\mathrm{InMe}_{3}$ dose (Figure S2 in the Supporting Information).

The exothermic reaction pathway for trivalent metal binding derived from theory predicts metal atom placement at highsymmetry nodal sites. Each atom is expected to bridge three oxygen atoms, two deriving from previously terminal $-\mathrm{OH}_{2}$ and $-\mathrm{OH}$ groups, and one deriving from bridging core oxygen atom functionality $(\mu-\mathrm{O}$ or previous $\mu-\mathrm{OH})$. Experimental confirmation of ALD metal installation location was obtained from X-ray pair distribution function (PDF) analyses. This method yields atomic scale structural information with crystallographic resolution at a length scale suitable for analysis of few-atom clusters like the $\mathrm{Zr}_{6}$ node. A differential data plot reveals the new pair correlations present after $\mathrm{InMe}_{3} \mathrm{AIM}$, Figure 5 .

The experimental differential PDF reflects the new atomatom distances formed upon In-loading; the distances are

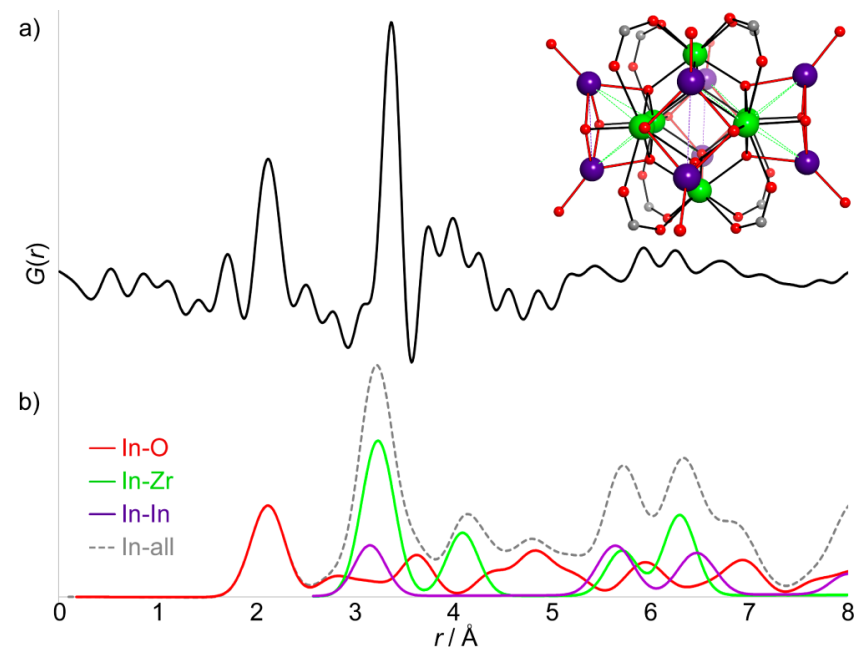

Figure 5. (a) Differential PDF corresponding to the new atom-atom distances formed upon $\mathrm{InMe}_{3} \mathrm{AIM}$ of NU-1000 at $75{ }^{\circ} \mathrm{C}$ following dehydration at $125^{\circ} \mathrm{C}$. (b) Partial pair PDFs calculated based on the model for In-loaded NU-1000 structure predicted by DFT. A representation of the DFT-derived model of the $\mathrm{Zr}_{6}$ node, highlighting the new In-node distances, is inset. 


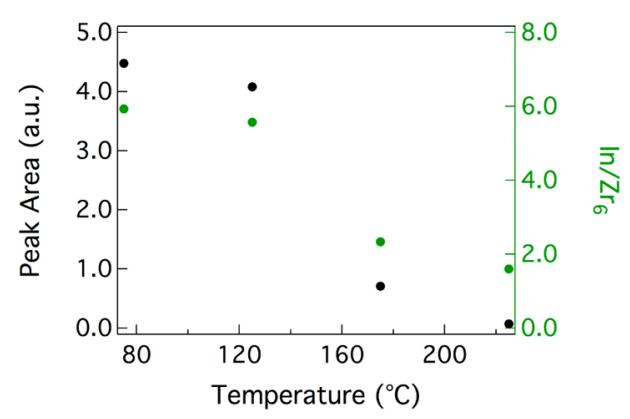

Figure 6. In-loading in NU-1000 as a function of node dehydration; temperature is correlated to the integrated intensity of FTIR peaks associated with $-\mathrm{OH}$ stretches.

consistent with those derived from first-principles calculations. The differential PDF is dominated by features at short distances, suggesting that the In-siting is well-defined only locally. Two sharp peaks, centered at 2.12 and $3.33 \AA$, correspond to the In-O bond and $\mathrm{In} \cdots \mathrm{Zr}$ next-nearest neighbor distances, respectively (cf. 2.18 and $3.27 \AA$ from theory, respectively). A broader feature at $\sim 4 \AA$ reflects longer range $\mathrm{In} \cdots \mathrm{Zr}$ distances within the $\mathrm{Zr}$-node, for which a distribution of distances and increased local disorder are evident. Peaks associated with well-defined In ‥In distances are not readily evident in the experimental data, suggesting that the In atoms do not order relative to each other.

Having established the location and bonding environment of In upon exposure to $\mathrm{InMe}_{3}$, we sought to further control the number of metal atoms installed on each node. In principle, the room temperature population of terminal $-\mathrm{OH}$, terminal $-\mathrm{OH}_{2}$, and $\mu-\mathrm{OH}$ on the NU-1000 node support the installation of up to 8 metal atoms. However, under conditions in which the node is dehydrated (e.g., following heating in medium vacuum), a diminished number of presenting functional groups is anticipated to yield a lower degree of metal incorporation under the same AIM conditions. Like many oxide surfaces, the $\mathrm{Zr}_{6} \mathrm{O}_{16}$ node releases water through water desorption and hydroxyl recombination at increased temperatures under vacuum. In situ Fourier transform infrared spectroscopy (conventional FTIR) studies of NU-1000 as a function of temperature clearly reveal dynamics of node dehydration. At elevated temperatures $\left(>125^{\circ} \mathrm{C}\right)$, the intensity of non-hydrogen-bonded $-\mathrm{OH},-\mathrm{OH}_{2}$, and $\mu-\mathrm{OH}$ stretches located at $\sim 3673 \mathrm{~cm}^{-1}$ decrease continuously with time and temperature (Figure S3 in the Supporting Information). On the basis of these findings, we performed node dehydration at elevated temperatures prior to AIM to provide control over the number of metals to be installed on the nodes. Although we do not expect a $1: 1$ correlation between $-\mathrm{OH}$ population as inferred from IR and the $\mathrm{In}: \mathrm{Zr}_{6}$ ratio, the integrated intensity of the $-\mathrm{OH}$ stretches (i.e., a measure of $-\mathrm{OH}$ population) and the ICP-OES derived ratios of In: $\mathrm{Zr}_{6}$ do trend together (Figure 6 ). This confirms an ability to control metal loading via node dehydration. Such ability to tune metal loading should allow further customization of node decoration, with the potential for mixed-metal attachment directly to the $\mathrm{Zr}_{6}$ node in the future through cycles of partial dehydration, deposition, rehydration, and alternative deposition.

\section{CONCLUSIONS}

We demonstrate that the $\mathrm{Zr}_{6}$-node present in the NU-1000 framework reacts with the trimethylated trivalent metal compounds $\mathrm{InMe}_{3}$ and $\mathrm{AlMe}_{3}$ to form stable structures with up to eight metals per $\mathrm{Zr}_{6}$ node. $\mathrm{InMe}_{3}$ exhibits strict selflimiting behavior in the limit of both excess diffusion time and moles without significant loss of crystallinity or internal surface area. Differential PDF analysis of In-loaded NU-1000 is consistent with theoretical prediction of a highly symmetric structure having two metals deposited on each of four faces of the $\mathrm{Zr}_{6}$ node; a fully characterized reaction coordinate rationalizes the elementary reaction steps that lead to this structure and confirms that it is highly exothermic. In practice, the number of In per $\mathrm{Zr}_{6}$ node saturates at a slightly less than 8:1 stoichiometric ratio, which we attribute to the presence of secondary nodes and unintentional dehydration, both of which reduce the total number of reactive sites. In contrast to $\mathrm{InMe}_{3}$, which displays ideal self-limiting behavior, reactions with $\mathrm{AlMe}_{3}$ evidence extra-nodal reactions with NU-1000 for large exposures, as judged by noticeable degradation of crystallinity and loss of internal surface area. These combined results begin to demarcate process variable space for AIM, i.e. temperature and choice of precursors, that must be carefully considered to achieve targeted metal loading while retaining the benefits of mesoporosity and crystallinity. We anticipate reporting soon on using these insights to achieve more ideal functionalization of NU-1000 with $\mathrm{Al}(\mathrm{III})$. Importantly, deliberate partial node dehydration at elevated AIM temperatures enables reproducible modulation of the stoichiometry for a single ALD cycle of the saturating reaction of $\mathrm{InMe}_{3}$ with NU-1000, and this control should prove useful for the design of multistep ALD procedures that may involve different metal-containing precursors.

\section{EXPERIMENTAL SECTION}

Computational Methods. The periodic structures were computed with the generalized gradient approximation exchangecorrelation functional PBE using the Vienna $a b$ initio simulation package (VASP). ${ }^{38-44}$ The VASP calculations were performed with projector-augmented wave potentials to describe the interaction between the valence and core electrons. A full gamma-point only geometry optimization was performed with a plane-wave kinetic energy cutoff of $520 \mathrm{eV}$. The energy and force convergence criteria were $1 \times 10^{-6}$ and $0.05 \mathrm{eV}$, respectively.

The initial structures for the cluster models were taken from ref 12 . The bare $\mathrm{Zr}_{6}$-node cluster model is provided in Figure 1. For the structures shown in the reaction pathway in Figure 2, the core zirconium and oxygen atoms were optimized with Gaussian $09^{45,46}$ using the M06-L ${ }^{47}$ density functional and the $6-31 \mathrm{G}(\mathrm{d})$ basis set $^{48}$ on $\mathrm{Al}, \mathrm{O}, \mathrm{C}$, and $\mathrm{H}$, and the Stuttgart/Dresden effective core potential (SDD) ${ }^{49}$ on $\mathrm{Zr}$ and $\mathrm{In}$, and the linker atoms were fixed. Frequency calculations at $298 \mathrm{~K}$ were then performed at the same level of theory. A singlepoint calculation with the $6-311+\mathrm{G}(\mathrm{df}, \mathrm{p})$ basis set ${ }^{48}$ was then performed to verify the nature of all stationary points. The enthalpies shown in Figure 2 were then computed by adding the enthalpy contribution at the $6-31 \mathrm{G}(\mathrm{d})$ level to the $6-311+\mathrm{G}(\mathrm{df}, \mathrm{p})$ energies $(\Delta H$ $\left.=\Delta E_{6-311+\mathrm{G}(\mathrm{df}, \mathrm{p})}+\Delta H_{6-31 \mathrm{G}(\mathrm{d})}-\Delta E_{6-31 \mathrm{G}(\mathrm{d})}\right)$.

NU-1000 Synthesis. $\mathrm{ZrOCl}_{2} \cdot 8 \mathrm{H}_{2} \mathrm{O}(97 \mathrm{mg}, 0.30 \mathrm{mmol})$ and benzoic acid $(2.70 \mathrm{~g}, 22 \mathrm{mmol}$ ) were mixed in $8 \mathrm{~mL}$ of DMF (in a 6dram vial) and dissolved using sonication. The clear solution was incubated in an oven at $80{ }^{\circ} \mathrm{C}$ for $1 \mathrm{~h}$. After cooling to room temperature, $\mathbf{H}_{4}$ TBAPy $(40 \mathrm{mg}, 0.06 \mathrm{mmol}$ ) was added to this solution and the mixture was sonicated for $10 \mathrm{~min}$. The yellow suspension was heated in an oven at $100{ }^{\circ} \mathrm{C}$ for $18 \mathrm{~h}$. After cooling to room temperature, yellow NU-1000 precipitate was collected by centrifuge. (7800 rpm, $5 \mathrm{~min}$ ) and washed with fresh DMF for two times. As synthesized NU-1000 was then suspended in $13 \mathrm{~mL}$ of DMF and $0.5 \mathrm{~mL}$ of $8 \mathrm{M} \mathrm{HCl}$ was added to acid-activate the zirconium node. This mixture was heated in an oven at $100{ }^{\circ} \mathrm{C}$ for $24 \mathrm{~h}$. After cooling down to room temperature, the supernatant was removed by 
centrifuge, and the material was washed twice with fresh DMF. Subsequently the solid materials were washed twice with acetone and soaked in acetone for additional $12 \mathrm{~h}$. Then the acetone was removed by centrifuge, and the solid was briefly dried in an oven at $80{ }^{\circ} \mathrm{C}$. The solid material was activated on a SmartVacPrep station (Micromeritics) under dynamic vacuum at $120^{\circ} \mathrm{C}$ until an outgassing rate of $\leq 0.002 \mathrm{mmHg} \mathrm{min}^{-1}$ was reached.

ALD in MOFs (AIM). AIM process was performed in a Savannah S200 system (Cambridge Nanotech, Cambridge, MA). In-line Entegris $\mathrm{Ni}$ filtered nitrogen $\left(\mathrm{N}_{2}\right)$ was continuously introduced to the ALD chamber at a flow rate of $20 \mathrm{sccm}$ to maintain a chamber base pressure of $\sim 0.5$ Torr. Typically, $10 \mathrm{mg}$ of NU-1000 powder was heated to 125 ${ }^{\circ} \mathrm{C}$ to remove physisorbed water followed by soaking at the desired AIM temperature. All ALD reactions were carried out in quasi-static mode. Standard pulse times for water vapor, $\mathrm{InMe}_{3}$, and $\mathrm{AlMe}_{3}$ were 1 , 5 , and $0.015 \mathrm{~s}$, respectively.

PDF Analysis. X-ray total scattering data suitable for PDF analysis were collected at beamline 11-ID-B at the Advanced Photon Source (APS). High-energy X-rays $(\lambda=0.2114 \AA)$ were used in combination with a large amorphous-silicon-based area detector to collect data to high values of momentum transfer $(Q) .^{50}$ Borosilicate capillary-loaded samples of NU-1000 and $\mathrm{InMe}_{3}$-AIM treated NU-1000 $\left(125{ }^{\circ} \mathrm{C}\right.$ dehydration followed by AIM at $75{ }^{\circ} \mathrm{C}$ followed by exposure to room ambient) were heated at $125^{\circ} \mathrm{C}$ under vacuum in a flow-cell furnace ${ }^{51}$ during the measurements. The two-dimensional X-ray scattering images were reduced to one-dimensional diffraction data within fit2D. The data were corrected for background, Compton scattering and detector effects within pdfgetX2 $2^{52}$ to obtain the structure function $S(Q)$. Fourier transformation of $S(Q)$ to $Q_{\max }=24 \AA^{-1}$, yielded the total PDFs, $G(r)$. Subtraction of the PDF for pristine NU-1000 from that obtained for $\mathrm{InMe}_{3}$-AIM treated NU-1000 yielded the differential $\mathrm{PDF}^{53}$ The bond lengths of features of interest were estimated by fitting Gaussian functions to the differential PDF data within fityk. ${ }^{54}$ For comparison with the experimental differential data, partial pair PDFs for the DFT-optimized model were calculated within PDFgui. ${ }^{55}$ The partial pair PDFs are given by $G(r)=(1 / r) \sum_{i} \sum_{j}\left[\left(\left(b_{i} b_{j}\right) /\right.\right.$ $\left.\left.\left(\langle b\rangle^{2}\right)\right) \delta\left(r-r_{i j}\right)\right]-4 \pi r \rho_{0}$, where the sum goes over pairs of atoms $i$ and $j$ within the model separated by $r_{i j}$. The scattering power of atom $i$ is $b_{i}$ and $\langle b\rangle$ is the average scattering power of the sample.

\section{ASSOCIATED CONTENT}

\section{S Supporting Information}

PXRD, BET, in situ FTIR data, predicted structures, and energetics for the 8:1 decorated nodes having either $\mathrm{CH}_{3}$ or $\mathrm{OH}$ groups on the Group 13 metal atoms, and the complete citation for ref 45. The Supporting Information is available free of charge on the ACS Publications website at DOI: 10.1021/ acs.chemmater.5b01560.

\section{AUTHOR INFORMATION}

\section{Corresponding Authors}

*E-mail: martinson@anl.gov.

*E-mail: cramer@umn.edu.

\section{Notes}

The authors declare no competing financial interest.

\section{ACKNOWLEDGMENTS}

This work was supported as part of the Inorganometallic Catalysis Design Center, an Energy Frontier Research Center funded by the U.S. Department of Energy, Office of Science, Basic Energy Sciences under Award DE-SC0012702. Work done at Argonne made use of the Advanced Photon Source, an Office of Science User Facility operated for the U.S. DOE/ Office of Science by Argonne National Laboratory, and was supported by the US DOE, Contract DE-AC02-06CH11357. We thank Dr. J. W. Elam and Dr. J. A. Libera for technical assistance and the use of their in situ FTIR system. We also thank Dr. L. M. Utschig-Johnson and Dr. S. R. Soltau for the use of their ICP-OES system. Finally, we thank Prof. D. G. Truhlar and Dr. L. Fernandez for helpful discussions.

\section{REFERENCES}

(1) Lee, J.; Farha, O. K.; Roberts, J.; Scheidt, K. A.; Nguyen, S. T.; Hupp, J. T. Metal-Organic Framework Materials as Catalysts. Chem. Soc. Rev. 2009, 38, 1450-1459.

(2) Ma, L.; Abney, C.; Lin, W. Enantioselective Catalysis with Homochiral Metal-Organic Frameworks. Chem. Soc. Rev. 2009, 38, $1248-1256$.

(3) Roy, S.; George, C. B.; Ratner, M. A. Catalysis by a ZincPorphyrin-Based Metal-Organic Framework: From Theory to Computational Design. J. Phys. Chem. C 2012, 116, 23494-23502.

(4) Planas, N.; Dzubak, A. L.; Poloni, R.; Lin, L.-C.; McManus, A.; McDonald, T. M.; Neaton, J. B.; Long, J. R.; Smit, B.; Gagliardi, L. The Mechanism of Carbon Dioxide Adsorption in an AlkylamineFunctionalized Metal-Organic Framework. J. Am. Chem. Soc. 2013, $135,7402-7405$.

(5) Oxford, G. A. E.; Dubbeldam, D.; Broadbelt, L. J.; Snurr, R. Q. Elucidating Steric Effects on Enantioselective Epoxidation Catalyzed by (Salen)Mn in Metal-Organic Frameworks. J. Mol. Catal. A: Chem. 2011, 334, 89-97.

(6) Odoh, S. O.; Cramer, C. J.; Truhlar, D. G.; Gagliardi, L. Quantum-Chemical Characterization of the Properties and Reactivities of Metal-Organic Frameworks. Chem. Rev. 2015, 6051-6111, DOI: $10.1021 / \mathrm{cr} 500551 \mathrm{~h}$.

(7) Mondloch, J. E.; Farha, O. K. and Hupp, J. T., Catalysis at the Organic Ligands. In Metal Organic Frameworks as Heterogeneous Catalysts; Xamena, F. L. i., Gascon, J., Eds.; The Royal Society of Chemistry: Cambridge, U.K., 2013; pp 289-309.

(8) Indeed, the earliest report of catalysis by a MOF material entails node catalysis. See: J. Am. Chem. Soc. 1994, 116, 1151.

(9) Vermoortele, F.; Valvekens, P. and De Vos, D., Catalysis at the Metallic Nodes of Mofs. In Metal Organic Frameworks as Heterogeneous Catalysts; Xamena, F. L. i., Gascon, J., Eds.; The Royal Society of Chemistry: Cambridge, U.K., 2013; pp 268-288.

(10) Corma, A.; Iglesias, M.; Llabrés i Xamena, F. X.; Sánchez, F. Cu and $\mathrm{Au}$ Metal-Organic Frameworks Bridge the Gap between Homogeneous and Heterogeneous Catalysts for Alkene Cyclopropanation Reactions. Chem.-Eur. J. 2010, 16, 9789-9795.

(11) Henschel, A.; Gedrich, K.; Kraehnert, R.; Kaskel, S. Catalytic Properties of MIL-101. Chem. Commun. 2008, 4192-4194, DOI: $10.1039 / \mathrm{B} 718371 \mathrm{~B}$.

(12) Beyzavi, M. H.; Klet, R. C.; Tussupbayev, S.; Borycz, J.; Vermeulen, N. A.; Cramer, C. J.; Stoddart, J. F.; Hupp, J. T.; Farha, O. K. A Hafnium-Based Metal-Organic Framework as an Efficient and Multifunctional Catalyst for Facile $\mathrm{CO}_{2}$ Fixation and Regioselective and Enantioretentive Epoxide Activation. J. Am. Chem. Soc. 2014, 136, 15861-15864.

(13) Katz, M. J.; Mondloch, J. E.; Totten, R. K.; Park, J. K.; Nguyen, S. T.; Farha, O. K.; Hupp, J. T. Simple and Compelling Biomimetic Metal-Organic Framework Catalyst for the Degradation of Nerve Agent Simulants. Angew. Chem. 2014, 126, 507-511.

(14) Mondloch, J. E.; Katz, M. J.; Isley Iii, W. C.; Ghosh, P.; Liao, P.; Bury, W.; Wagner, G. W.; Hall, M. G.; DeCoste, J. B.; Peterson, G. W.; et al. Destruction of Chemical Warfare Agents Using Metal-Organic Frameworks. Nat. Mater. 2015, 14, 512-516.

(15) Vermoortele, F.; Vandichel, M.; Van de Voorde, B.; Ameloot, R; Waroquier, M.; Van Speybroeck, V.; De Vos, D. E. Electronic Effects of Linker Substitution on Lewis Acid Catalysis with MetalOrganic Frameworks. Angew. Chem., Int. Ed. 2012, 51, 4887-4890.

(16) Phan, A.; Czaja, A. U.; Gándara, F.; Knobler, C. B.; Yaghi, O. M. Metal-Organic Frameworks of Vanadium as Catalysts for Conversion of Methane to Acetic Acid. Inorg. Chem. 2011, 50, 7388-7390.

(17) Nguyen, H. G. T.; Schweitzer, N. M.; Chang, C.-Y.; Drake, T. L.; So, M. C.; Stair, P. C.; Farha, O. K.; Hupp, J. T.; Nguyen, S. T. 
Vanadium-Node-Functionalized UiO-66: A Thermally Stable MofSupported Catalyst for the Gas-Phase Oxidative Dehydrogenation of Cyclohexene. ACS Catal. 2014, 4, 2496-2500.

(18) Deria, P.; Mondloch, J. E.; Tylianakis, E.; Ghosh, P.; Bury, W.; Snurr, R. Q.; Hupp, J. T.; Farha, O. K. Perfluoroalkane Functionalization of NU-1000 Via Solvent-Assisted Ligand Incorporation: Synthesis and $\mathrm{Co}_{2}$ Adsorption Studies. J. Am. Chem. Soc. 2013, 135, 16801-16804.

(19) Deria, P.; Bury, W.; Hupp, J. T.; Farha, O. K. Versatile Functionalization of the NU-1000 Platform by Solvent-Assisted Ligand Incorporation. Chem. Commun. 2014, 50, 1965-1968.

(20) Deria, P.; Bury, W.; Hod, I.; Kung, C.-W.; Karagiaridi, O.; Hupp, J. T.; Farha, O. K. MOF Functionalization via Solvent-Assisted Ligand Incorporation: Phosphonates vs Carboxylates. Inorg. Chem. 2015, 54, $2185-2192$.

(21) Hod, I.; Bury, W.; Gardner, D. M.; Deria, P.; Roznyatovskiy, V.; Wasielewski, M. R.; Farha, O. K.; Hupp, J. T. Bias-Switchable Permselectivity and Redox Catalytic Activity of a Ferrocene-Functionalized, Thin-Film Metal-Organic Framework Compound. J. Phys. Chem. Lett. 2015, 6, 586-591.

(22) Deria, P.; Mondloch, J. E.; Karagiaridi, O.; Bury, W.; Hupp, J. T.; Farha, O. K. Beyond Post-Synthesis Modification: Evolution of Metal-Organic Frameworks Via Building Block Replacement. Chem. Soc. Rev. 2014, 43, 5896-5912.

(23) Ritala, M. and Niinisto, J., Atomic Layer Deposition. In Chemical Vapour Deposition: Precursors, Processes and Applications; Jones, A. C., Hitchman, M. L., Eds.; The Royal Society of Chemistry: Cambridge, U.K., 2009.

(24) George, S. M. Atomic Layer Deposition: An Overview. Chem. Rev. 2010, 110, 111-131.

(25) Leskelä, M.; Ritala, M. Atomic Layer Deposition (ALD): From Precursors to Thin Film Structures. Thin Solid Films 2002, 409, 138146.

(26) Mondloch, J. E.; Bury, W.; Fairen-Jimenez, D.; Kwon, S.; DeMarco, E. J.; Weston, M. H.; Sarjeant, A. A.; Nguyen, S. T.; Stair, P. C.; Snurr, R. Q.; et al. Vapor-Phase Metalation by Atomic Layer Deposition in a Metal-Organic Framework. J. Am. Chem. Soc. 2013, 135, 10294-10297.

(27) Cavka, J. H.; Jakobsen, S.; Olsbye, U.; Guillou, N.; Lamberti, C.; Bordiga, S.; Lillerud, K. P. A New Zirconium Inorganic Building Brick Forming Metal Organic Frameworks with Exceptional Stability. J. Am. Chem. Soc. 2008, 130, 13850-13851.

(28) Groner, M. D.; Fabreguette, F. H.; Elam, J. W.; George, S. M. Low-Temperature $\mathrm{Al}_{2} \mathrm{O}_{3}$ Atomic Layer Deposition. Chem. Mater. 2004, 16, 639-645.

(29) Heil, S. B. S.; Langereis, E.; Roozeboom, F.; van de Sanden, M. C. M.; Kessels, W. M. M. Low-Temperature Deposition of Tin by Plasma-Assisted Atomic Layer Deposition. J. Electrochem. Soc. 2006, 153, G956-G965.

(30) Bakke, J. R.; King, J. S.; Jung, H. J.; Sinclair, R.; Bent, S. F. Atomic Layer Deposition of $\mathrm{ZnS}$ via in situ Production of $\mathrm{H}_{2} \mathrm{~S}$. Thin Solid Films 2010, 518, 5400-5408.

(31) Ten Eyck, G. A.; Senkevich, J. J.; Tang, F.; Liu, D.; Pimanpang, S.; Karaback, T.; Wang, G. C.; Lu, T. M.; Jezewski, C.; Lanford, W. A. Plasma-Assisted Atomic Layer Deposition of Palladium. Chem. Vapor Depos. 2005, 11, 60-66.

(32) Miikkulainen, V.; Leskelä, M.; Ritala, M.; Puurunen, R. L. Crystallinity of Inorganic Films Grown by Atomic Layer Deposition: Overview and General Trends. J. Appl. Phys. 2013, 113, 021301.

(33) Planas, N.; Mondloch, J. E.; Tussupbayev, S.; Borycz, J.; Gagliardi, L.; Hupp, J. T.; Farha, O. K.; Cramer, C. J. Defining the Proton Topology of the $\mathrm{Zr}_{6}$-Based Metal-Organic Framework NU1000. J. Phys. Chem. Lett. 2014, 5, 3716-3723.

(34) Goldstein, D. N.; McCormick, J. A.; George, S. M. $\mathrm{Al}_{2} \mathrm{O}_{3}$ Atomic Layer Deposition with Trimethylaluminum and Ozone Studied by in Situ Transmission Ftir Spectroscopy and Quadrupole Mass Spectrometry. J. Phys. Chem. C 2008, 112, 19530-19539.

(35) Elam, J. W.; Routkevitch, D.; Mardilovich, P. P.; George, S. M. Conformal Coating on Ultrahigh-Aspect-Ratio Nanopores of Anodic
Alumina by Atomic Layer Deposition. Chem. Mater. 2003, 15, 35073517.

(36) Hinkle, C. L.; Sonnet, A. M.; Vogel, E. M.; McDonnell, S.; Hughes, G. J.; Milojevic, M.; Lee, B.; Aguirre-Tostado, F. S.; Choi, K. J.; Kim, H. C.; et al. GaAs Interfacial Self-Cleaning by Atomic Layer Deposition. Appl. Phys. Lett. 2008, 92, 071901.

(37) Milojevic, M.; Aguirre-Tostado, F. S.; Hinkle, C. L.; Kim, H. C.; Vogel, E. M.; Kim, J.; Wallace, R. M. Half-Cycle Atomic Layer Deposition Reaction Studies of $\mathrm{Al}_{2} \mathrm{O}_{3}$ on $\mathrm{In}_{0.2} \mathrm{Ga}_{0.8} \mathrm{As}$ (100) Surfaces. Appl. Phys. Lett. 2008, 93, 202902.

(38) Perdew, J. P.; Wang, Y. Accurate and Simple Analytic Representation of the Electron-Gas Correlation Energy. Phys. Rev. B 1992, 45, 13244-13249.

(39) Perdew, J. P.; Burke, K.; Ernzerhof, M. Generalized Gradient Approximation Made Simple. Phys. Rev. Lett. 1996, 77, 3865-3868.

(40) Perdew, J. P.; Ernzerhof, M.; Burke, K. Rationale for Mixing Exact Exchange with Density Functional Approximations. J. Chem. Phys. 1996, 105, 9982-9985.

(41) Kresse, G.; Furthmüller, J. Efficient Iterative Schemes for $\mathrm{Ab}$ Initio Total-Energy Calculations Using a Plane-Wave Basis Set. Phys. Rev. B 1996, 54, 11169-11186.

(42) Kresse, G.; Furthmüller, J. Efficiency of Ab-Initio Total Energy Calculations for Metals and Semiconductors Using a Plane-Wave Basis Set. Comput. Mater. Sci. 1996, 6, 15-50.

(43) Kresse, G.; Hafner, J. Ab Initio Molecular-Dynamics Simulation of the Liquid-Metal-Amorphous-Semiconductor Transition in Germanium. Phys. Rev. B 1994, 49, 14251-14269.

(44) Kresse, G.; Hafner, J. Ab Initio Molecular Dynamics for Liquid Metals. Phys. Rev. B 1993, 47, 558-561.

(45) Frisch, M. J.; Trucks, G. W.; Schlegel, H. B.; Scuseria, G. E.; Robb, M. A.; Cheeseman, J. R.; Scalmani, G.; Barone, V.; Mennucci, B.; Petersson, G. A.; et al. Gaussian 09, Revisions A.02, B.01 and C.01; Gaussian Inc.: Wallinfgord, CT, 2009.

(46) Zhao, Y.; Peverari, R.; Yang, K. and Truhlar, D. G. MN-GFM, 6.4; University of Minnesota: Minneapolis, MN, 2012.

(47) Zhao, Y.; Truhlar, D. G. A New Local Density Functional for Main-Group Thermochemistry, Transition Metal Bonding, Thermochemical Kinetics, and Noncovalent Interactions. J. Chem. Phys. 2006, 125, 194101.

(48) Hehre, W. J.; Radom, L.; Schleyer, P. v. R. and Pople, J. A. Ab Initio Molecular Orbital Theory; Wiley: New York, 1986.

(49) Nicklass, A.; Dolg, M.; Stoll, H.; Preuss, H. Ab Initio EnergyAdjusted Pseudopotentials for the Noble Gases Ne through Xe: Calculation of Atomic Dipole and Quadrupole Polarizabilities. J. Chem. Phys. 1995, 102, 8942-8952.

(50) Chupas, P. J.; Lee, P. L.; Chapman, K. W. Applications of an Amorphous Silicon-Based Area Detector for High-Resolution, HighSensitivity and Fast Time-Resolved Pair Distribution Function Measurements. J. Appl. Crystallogr. 2007, 40, 463-470.

(51) Chupas, P. J.; Chapman, K. W.; Kurtz, C.; Hanson, J. C.; Lee, P. L.; Grey, C. P. A Versatile Sample-Environment Cell for Non-Ambient X-Ray Scattering Experiments. J. Appl. Crystallogr. 2008, 41, 822-824.

(52) Qiu, X.; Thompson, J. W.; Billinge, S. J. L. PDFgetX2: A GuiDriven Program to Obtain the Pair Distribution Function from X-Ray Powder Diffraction Data. J. Appl. Crystallogr. 2004, 37, 678-678.

(53) Chapman, K. W.; Chupas, P. J.; Kepert, C. J. Selective Recovery of Dynamic Guest Structure in a Nanoporous Prussian Blue through in Situ X-Ray Diffraction: A Differential Pair Distribution Function Analysis. J. Am. Chem. Soc. 2005, 127, 11232-11233.

(54) Wojdyr, M. Fityk: A General-Purpose Peak Fitting Program. J. Appl. Crystallogr. 2010, 43, 1126-1128.

(55) Farrow, C. L.; Juhas, P.; Liu, J. W.; Bryndin, D.; Božin, E. S.; Bloch, J.; Th, P.; Billinge, S. J. L. PDFfit2 and PDFgui: Computer Programs for Studying Nanostructure in Crystals. J. Phys.: Condens. Matter 2007, 19, 335219. 